\title{
Olmesartan induced gastroenteropathy and acute kidney injury: A case report and review of literature
}

\author{
Vani Thirumala, Miller Timothy, Seshadri Thirumala
}

\begin{abstract}
Olmesartan, an Angiotensin-Receptor blocker (ARB) has been widely reported as causing severe enteropathy. We present a case of Acute Kidney Injury (AKI) secondary to severe diarrhea induced by Olmesartan therapy in a 58-year-old patient. Acute kidney injury (AKI) is an abrupt and usually reversible decline in the glomerular filtration rate (GFR). This results in an elevation of serum blood urea nitrogen (BUN), creatinine, and other metabolic waste products that are normally excreted by the kidney. Clinical presentation of acute kidney injury in patients on Olmesartan can be misleading and delay accurate diagnosis and treatment.
\end{abstract}

Keywords: Acute kidney injury, Angiotensin converting enzyme, Angitotensin receptor blocker, Enteropathy, Olmesartan, Sprue

\section{How to cite this article}

Thirumala V, Timothy M, Thirumala S. Olmesartan induced gastroenteropathy and acute kidney injury: A case report and review of literature. J Case Rep Images Pathol 2018;4:100019Z11VT2018.

Vani Thirumala ${ }^{1}$, Miller Timothy ${ }^{2}$, Seshadri Thirumala ${ }^{3}$

Affiliations: ${ }^{1} B S A$ Neurosciences, Pre Med Student, UT Austin, Texas, USA; ${ }^{2} \mathrm{MD}$ Gastroenterology, LDDA, Lubbock Texas, USA; ${ }^{3} \mathrm{MD}$ Surgical Pathology, Ameripath Lubbock, Texas, USA.

Corresponding Author: Seshadri Thirumala, MD, 1912 Aberdeen Ave, Lubbock, Texas 79407, USA; Email: seshathiru@yahoo.com

Received: 31 January 2017

Accepted: 27 February 2018

Published: 12 March 2018
Article ID: 100019Z11VT2018

$* * * * * * * * *$

doi: 10.5348/100019Z11VT2018CR

\section{INTRODUCTION}

Olmesartan, an Angiotensin-Receptor blocker (ARB) has been widely reported as causing severe enteropathy. We present a case of Acute Kidney Injury (AKI) secondary to severe diarrhea induced by olmesartan therapy in a 58-year-old patient. Olmesartan is a selective and competitive, non-peptide angiotensin II receptor antagonist widely used in treating patients with hypertension [1]. Other uses of this drug include prevention of microalbuminuria in Type II Diabetes, acute coronary syndrome/secondary prevention of cardiovascular events and to improve kidney outcomes in hypertensive patients with Chronic Kidney Disease [2]. Olmesartan blocks the vasoconstrictor and aldosterone secreting effects of angiotensin II. Unlike ACE inhibitors, ARBs induce more and sometimes complete inhibition of the renin-angiotensin system, and are less likely to be associated with side effects like cough and angioedema.

Classic celiac disease is a gluten sensitive enteropathy characterized by mucosal inflammation, villous atrophy and crypt hyperplasia. In adults celiac disease is most often diagnosed in the third and fourth decades of life, but may develop at any age [3]. Many adult patients present with diarrhea and weight loss. Since the introduction of highly sensitive autoantibody tests, the number of patients presenting with classic symptoms has decreased and atypical presenting signs including iron deficiency anemia are on the rise. Other atypical clinical presentation include vague abdominal pain, infertility, amenorrhea, peripheral neuropathy, ataxia and seizures [4]. Several other etiologic conditions including medications and drugs such as olmesartan, morbid obesity are described to mimic gluten sensitive enteropathy histologically and 


\section{EDORIUM Journals}

www.edoriumjournals.com/case-reports/jcrp

clinically. It is important to identify these entities and risk factors as management is different. Olmesartan is an angiotensin II receptor agonist used in the management of hypertension has been shown in several reports to induce 'sprue like enteropathy'. Persistent diarrhea due to enteropathy induced by the drug with resultant dehydration and hypovolemia could lead to acute kidney injury and renal failure [5, 6]. We present a case of olmesartan associated enteropathy presenting with manifestations of AKI secondary to hypovolemia and dehydration. The literature is reviewed and mechanisms of action and pitfalls discussed.

\section{CASE REPORT}

A 58-year-old man previously in good health presented to ER with chief complaints of weakness, abdominal pain, nausea, vomiting and diarrhea. He had not been able to eat much for several weeks due to profuse watery diarrhea with a $20 \mathrm{lbs}$ weight loss. He had a previous admission five weeks ago for similar complaints and was discharged on antibiotics. Colonoscopy performed at the initial admission revealed a benign hyperplastic polyp in the sigmoid colon. His symptoms worsened and he has been unable to stay hydrated due to severe diarrhea. A CT scan of the abdomen revealed non-specific enteritis. PCR test for Clostridium difficile and stool cultures were negative. Serologic studies for cryptosporidium and giardia were negative. Stool examination for parasites was negative and patient denied history of any travel. His erythrocyte sedimentation rate (ESR) at the time of admission was $3 \mathrm{ml} / \mathrm{hr}$ (ref. $\mathrm{O}-22 \mathrm{ml} / \mathrm{hr}$ ) and c-reactive protein (CRP) was $5.3 \mathrm{mg} / \mathrm{L}$ (ref. $<3.0 \mathrm{mg} / \mathrm{L}$ ). At the time of his initial admission five weeks ago, the estimated glomerular filtration rate (GFR) was $109 \mathrm{ml} / \mathrm{min}$ (ref $>60 \mathrm{ml} / \mathrm{min}$ ) with a creatinine level of $0.90 \mathrm{mg} / \mathrm{dl}$ (ref o.6-1.3 mg/dl). On second admission, he was found to have AKI with a creatinine of $5.4 \mathrm{mg} / \mathrm{dl}$ and his estimated GFR was $30 \mathrm{ml} / \mathrm{min}$. Ultra sound examination of kidneys showed no evidence of hydronephrosis or nephrolithiasis and no other acute findings. Patient denied any personal or family history of kidney dysfunction. He denied any recent fever, headache, cough, chest pain or shortness of breath. He had no previous history of chronic renal failure.

Biopsies were obtained during esophagogastroduodenoscopy (EGD) and Colonoscopy. Examination of duodenal (Figure 1), gastric (Figure 2), ileal (Figure 3) and colonic biopsies revealed marked lymphocytosis of the surface epithelium. In addition, duodenal biopsies showed villous blunting with marked crypt hyperplasia simulating celiac disease. Colon biopsies revealed features of collagenous colitis. Serological studies for celiac disease were negative and the patient denied any travel history. Genetic testing for celiac disease was not performed. His medication history included olmesartan 40mg daily for hypertension for the last two years prior to the onset of current symptoms. He has not been on any other medications except multivitamins. His symptoms resolved within two weeks of discontinuation of the therapy including gradual normalization of renal function tests and cessation of diarrhea. The patient

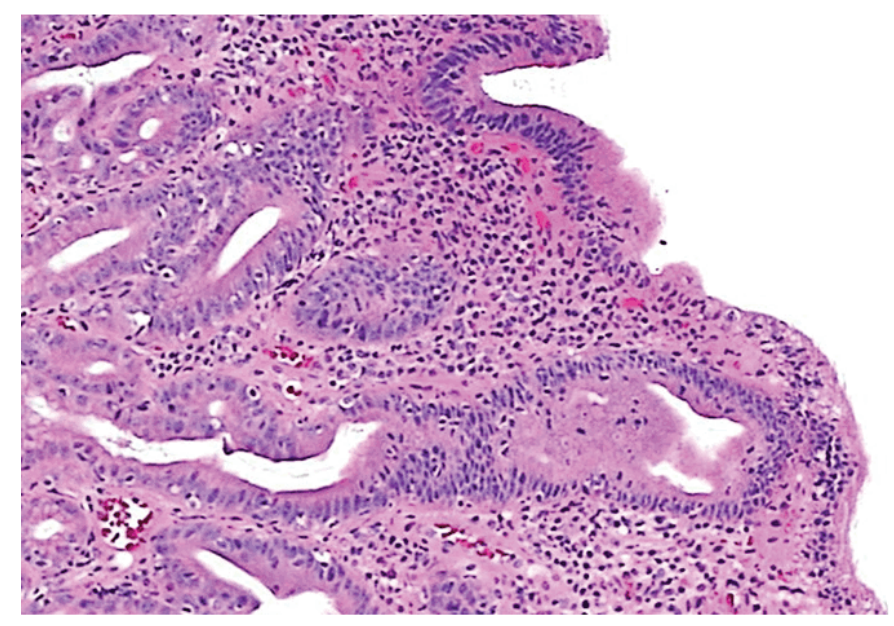

Figure 1: Duodenum biopsy showing villous blunting and increased intraepithelial lymphocytes (H\&E stain, x400).

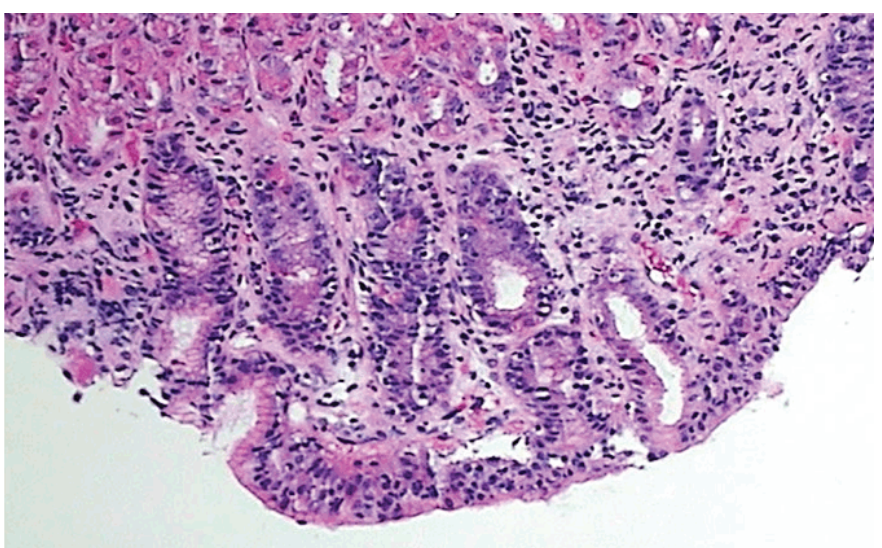

Figure 2: Gastric biopsy showing intraepithelial lymphocytosis (H\&E stain, x400).

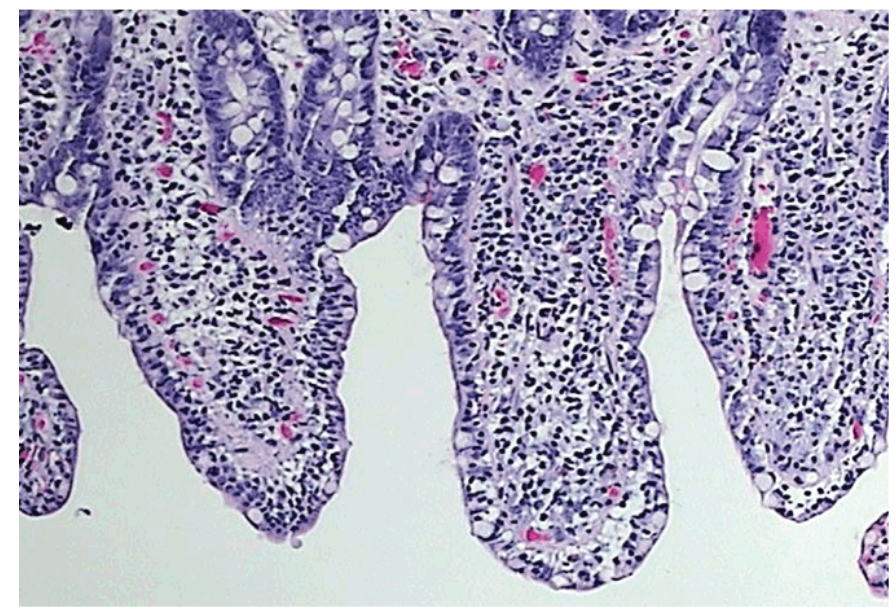

Figure 3: Ileal biopsy showing intraepithelial lymphocytosis (H\&E stain, x400). 


\section{EDORIUM Journals}

regained his weight in six weeks and his repeat biopsies were normal.

\section{DISCUSSION}

Rubio-Tapia et al [7] in 2012 described severe spruelike enteropathy secondary to olmesartan in a series of 22 patients. Serologic testing for celiac disease was negative in all cases and none improved with gluten free diet. Fifteen (15) of the twenty two (22) patients in their study showed villous atrophy with concomitant lymphocytosis in fourteen (14) of them. All these patients improved on discontinuation of olmesartan.

Lagana et al. [8] in a large retrospective study spanning over 10 years identified a total of 72 patients with seronegative villous atrophy at their institution. Seronegative celiac disease was diagnosed based on HLA typing in 20 of these patients and all responded to gluten free diet. Sixteen (16) of the Seventy two (72) patients were on olmesartan and these patients achieved complete clinical and histological remission on discontinuation of the drug. One (1) patient in their study presented with constipation rather than diarrhea and showed partial villous blunting which responded after stopping olmesartan. In this study, the authors observed a spectrum of changes in duodenal biopsies including crypt apoptosis, increased intraepithelial lymphocytosis and partial to complete villous blunting very similar to intestinal damage caused by mycophenolate. Intriguingly, their study found the histologic changes specific for olmesartan and not identified in those patients taking other ARBs. Further studies on this curious observation need to be done to confirm or refute their findings.

Several similar case series and case reports have been published in English language literature. DeGaetani et al. [9] in 2013 reported 16 cases, Burbure et al. [10] reported 72 cases with similar observations and temporal relationship between olmesartan and enteropathy.

Laniro et al. [11] in a systematic review of olmesartan associated sprue-like enteropathy observed that almost all of 54 patients in their study presented with diarrhea and weight loss. Normocytic Normochromic anemia and hypoalbuminemia were the most common findings observed in these patients. Celiac serologies were negative in all these patients. Withdrawal of olmesartan caused improvement of clinical symptoms, and histological remission in all of them establishing a temporal relationship of olmesartan to sprue-like enteropathy.

Several other entities have been described to cause increased intraepithelial lymphocytosis, villous blunting of duodenal mucosa causing malabsorption and diarrhea. These include but not limited to are, celiac disease, drugs including NSAIDs, autoimmune enteropathy, Crohn disease, giardiasis, small intestinal bacterial overgrowth (SIBO) morbid obesity etc. Celiac disease must be ruled out in any patient with villous blunting. Testing for anti- tissue Glutamase (tTG) is highly specific and essentially excludes celiac disease. Several drugs including olmesartan, valsartan, (ARBs) and immunosuppressive agents such as ipilimumab, mycophenolate, azathioprine, methotrexate can induce changes mimicking celiac disease on histology.

Olmesartan, in addition to causing villous blunting often has been reported to cause lymphocytic/collagenous gastritis and colitis. The terminal ileum biopsy in our patient exhibited marked increase in intraepithelial lymphocytes (Figure 3), however, villous blunting or collagen deposition were not observed.

Though, the exact mechanism of olmesartan induced lymphocyticgastro-enteropathy is not known, it is believed to be a cell-mediated immune response. Marietta et al. [11] proposed role for IL-15 signaling and disruption of the tight junction protein ZO-1 in disease pathogenesis. They concluded that olmesartan induced enteropathy shared the immunologic pathways with celiac disease, including recruitment of $\mathrm{CD} 8+\mathrm{T}$ cells with overexpression of IL- 5 by epithelial cells. In an another investigational study, Laniro et al. [10] speculated that villous atrophy might be the result of a pro-apoptotic effect of Angiotensin II on intestinal epithelial cells. Olmesartan and other ARBs have been shown to express high affinity for AT1 receptors in the gut epithelium, saturating them while Angiotensin II binds to free AT2 receptors. AT2 receptors, upon binding to Angiotensin II, exhibit a strong pro-apoptotic effect on intestinal epithelial cells, leading to increased IELs and villous atrophy.

Acute Kidney Injury (AKI), is the term used to describe an acute deterioration of renal function that has been present for less than three months. Patients with AKI usually present with symptoms and signs resulting directly from diminished kidney function which include edema, decreased urine output or, in severe cases anuria [12]. However, many patients have no clinical symptoms, and an increase in creatinine is detected by laboratory tests that are routinely obtained among hospitalized patients is the first indication of AKI. Etiologic factors are multiple and include pre-renal, renal and post renal causes. Acute Tubular Necrosis accounts for almost half of the cases of AKI [13]. Most common etiologies of for acute tubular necrosis include acute hemorrhage, severe diarrhea, sepsis and drug nephrotoxicity. Renal etiologies include intrinsic vascular, glomerular and tubule-interstitial disorders [14]. Post renal causes of AKI are mostly obstructive in nature and usually bilateral including prostatic hypertrophy and retroperitoneal fibrosis. Other non-renal causes of AKI include collagenous/lymphocytic colitis, celiac disease, infectious gastroenteritis by way of causing profuse watery diarrhea leading to volume depletion and renal failure. Collagenous colitis is characterized by sub-epithelial collagen band $>10$ micrometers in diameter [15]. The band is most evident between the crypts and often duplicating around 


\section{EDORIUM Journals}

capillaries. Lymphocytic colitis is characterized by $\geq 20$ intraepithelial lymphocytes (IEL) per 100 surface epithelial cells. Crypt architecture is usually not distorted, but focal cryptitis may be present.

Persistent diarrhea secondary to villous atrophy induced by olmesartan potentially can lead to AKI due to volume depletion. Few case reports of AKI secondary to profuse diarrheas have been reported in English language literature $[5,6]$. The largest series was reported by Lourdes-Roca-Argente et al. in the form of letter to editor. In their experience, acute renal failure secondary to olmesartan therapy was noted in 14 of 19 patients [16]. All the 14 patients in their study developed AKI secondary to volume depletion reverted to normal after rehydration and discontinuing olmesartan. None of their patients had any pre-existing renal conditions to explain AKI. In the current study, our patient also demonstrated complete clinical and histologic recovery after discontinuation of olmesartan therapy.

\section{CONCLUSION}

Acute Kidney Injury (AKI) was the presenting clinical feature in the current study. Thorough clinical and imaging studies evaluation revealed no significant renal and post renal causes to account for AKI in this patient. AKI was secondary to profuse diarrhea and fluid loss in this patient secondary to olmesartan and complete clinical and histologic recovery was demonstrated after discontinuing the drug. Possibility of and exclusion of infectious etiologies and de-novo collagenous/ lymphocytic colitis should always be considered in the differential diagnosis of profuse watery diarrhea. Critical review of clinical information including medication history and awareness of etiologic association between olmesartan and enteropathy is key to avoiding a potential pitfall of misdiagnosis and mismanagement.

\section{REFERENCES}

1. Burbure N, Lebwohl B, Arguelles-Grande C, Green PH, Bhagat G, Lagana S. Olmesartan-associated spruelike enteropathy: A systematic review with emphasis on histopathology. Hum Pathol 2016 Apr;50:127-34.

2. Haller H, Ito $\mathrm{S}$, Izzo JL Jr, et al. Olmesartan for the delay or prevention of microalbuminuria in type 2 diabetes. N Engl J Med 2011 Mar 10;364(10):907-17.

3. Hankey GL, Holmes GK. Coeliac disease in the elderly. Gut 1994 Jan;35(1):65-7.

4. Collin P, Vilska S, Heinonen PK, Hällström O, Pikkarainen P. Infertility and coeliac disease. Gut 1996 Sep;39(3):382-4.

5. Ulla-Rocha JL, Lopez-Piñeiro S, Puga-Gimenez M. Acute renal failure secondary to diarrhea due to sprue like-enteropathy associated with olmesartan. [Article in Spanish]. Gastroenterol Hepatol 2015 Oct;38(8):514-5.
6. Koizumi T, Furuya K, Baba M, Sadaoka K, Sekiya C, Hattori A. Case Report; Olmesartan associated enteropathy: A case of severe watery diarrhea with weight loss and acute renal failure. [Article in Japanese]. Nihon Naika Gakkai Zasshi 2015 Jun 10;104(6):1167-72.

7. Rubio-Tapia A, Herman ML, Ludvigsson JF, et al. Severe spruelike enteropathy associated with olmesartan. Mayo Clin Proc 2012 Aug;87(8):732-8.

8. Lagana SM, Braunstein ED, Arguelles-Grande C, Bhagat G, Green PH, Lebwohl B. Sprue-like histology in patients with abdominal pain taking olmesartan compared with other angiotensin receptor blockers. J Clin Pathol 2015 Jan;68(1):29-32.

9. DeGaetani M, Tennyson CA, Lebwohl B, et al. Villous atrophy and negative celiac serology: A diagnostic and therapeutic dilemma. Am J Gastroenterol 2013 May;108(5):647-53.

10. Ianiro G, Bibbò S, Montalto M, Ricci R, Gasbarrini A, Cammarota G. Systematic review: Sprue-like enteropathy associated with olmesartan. Aliment Pharmacol Ther 2014 Jul;40(1):16-23.

11. Marietta EV, Nadeau AM, Cartee AK, et al. Immunopathogenesis of olmesartan-associated enteropathy. Aliment Pharmacol Ther 2015 Dec;42(11-12):1303-14.

12. Balasubramanian G, Al-Aly Z, Moiz A, et al. Early nephrologist involvement in hospital-acquired acute kidney injury: A pilot study. Am J Kidney Dis 2011 Feb;57(2):228-34.

13. Liaño F, Pascual J. Epidemiology of acute renal failure: A prospective, multicenter, community-based study. Madrid acute renal failure study group. Kidney Int 1996 Sep;50(3):811-8.

14. Esson ML, Schrier RW. Diagnosis and treatment of acute tubular necrosis. Ann Intern Med 2002 Nov 5;137(9):744-52.

15. Veress B, Löfberg R, Bergman L. Microscopic colitis syndrome. Gut 1995 Jun;36(6):880-6.

16. Roca-Argente L, Diaz-Jaime FC, López-Romero LC, et al. Acute kidney injury secondary to diarrhea caused by "sprue-like" enteropathy associated with olmesartan. [Article in Spanish]. Nefrologia 2017 Sep-Oct;37(5):548-50.

$* * * * * * * * *$

\section{Author Contributions}

Vani Thirumala - Substantial contributions to conception and design, Acquisition of data, Analysis and Interpretation of data, Drafting of the article, Revising it critically for important intellectual content, Final approval of the version to be published

Miller Timothy - Substantial contributions to conception and design, Acquisition of data, Analysis and Interpretation of data, Drafting of the article, Revising it critically for important intellectual content, Final approval of the version to be published

Seshadri Thirumala - Substantial contributions to conception and design, Acquisition of data, Analysis and Interpretation of data, Drafting of the article, Revising it critically for important intellectual content, Final approval of the version to be published 


\section{EDORIUM Journals}

J Case Rep Images Pathol 2018;4:100019Z11VT2018.

Thirumala et al. 5

www.edoriumjournals.com/case-reports/jcrp

\section{Guarantor of Submission}

The corresponding author is the guarantor of submission.

\section{Source of Support}

None

\section{Consent Statement}

Written informed consent was obtained from the patient for publication of this study.

\section{Conflict of Interest}

Authors declare no conflict of interest.

\section{Copyright}

(C) 2018 Vani Thirumala et al. This article is distributed under the terms of Creative Commons Attribution License which permits unrestricted use, distribution and reproduction in any medium provided the original author(s) and original publisher are properly credited. Please see the copyright policy on the journal website for more information.
Access full text article on other devices

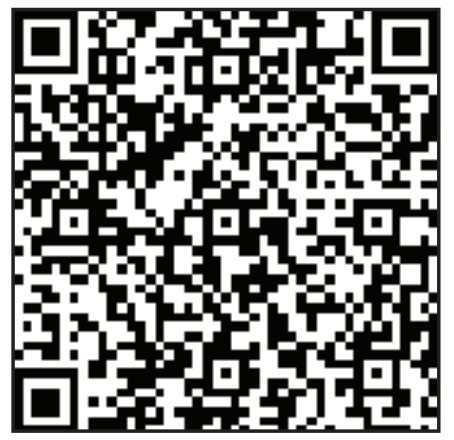

Access PDF of article on other devices

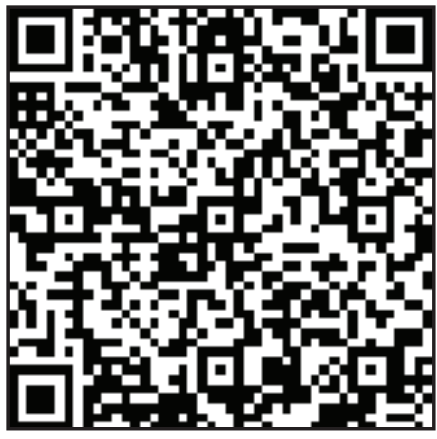

